\title{
Increased incidence of inflammatory bowel disease in Québec residents with airway diseases
}

\author{
Paul Brassard ${ }^{1,2}$, Maria Vutcovici ${ }^{3}$, Pierre Ernst ${ }^{1,2}$, Valérie Patenaude ${ }^{1}$, \\ Maida Sewitch ${ }^{2,4}$, Samy Suissa ${ }^{1,2}$ and Alain Bitton ${ }^{2,3}$
}

Affiliations: ${ }^{1}$ Centre for Clinical Epidemiology, Lady Davis Institute for Medical Research, Jewish General Hospital, Montreal, QC, Canada. ${ }^{2}$ McGill University, Faculty of Medicine, Montreal, QC, Canada. ${ }^{3}$ McGill University Health Centre, Division of Gastroenterology, Montreal, QC, Canada. ${ }^{4}$ McGill University Health Centre, Division of Clinical Epidemiology, Montreal, QC, Canada.

Correspondence: Paul Brassard, Centre for Clinical Epidemiology, Lady Davis Institute for Medical Research, Jewish General Hospital, 3755 Chemin de la Cote Ste-Catherine, H3T 1E2, Montréal, QC, Canada. E-mail: paul.brassardamcgill.ca

ABSTRACT The objective of the study was to assess whether the incidences of Crohn's disease and ulcerative colitis are increased in patients with asthma and chronic obstructive pulmonary disease (COPD) compared to the general population.

A population-based retrospective cohort study was conducted using the administrative health databases of Québec, Canada. Incidences of Crohn's disease and ulcerative colitis among patients with asthma and COPD were assessed for the 2001-2006 period.

In total, 136178 subjects with asthma and 143904 subjects with COPD were identified. The average incidence of Crohn's disease and ulcerative colitis was 23.1 and 8.8 per 100000 person-years among asthmatic patients; in the COPD cohort there were 26.2 Crohn's disease cases and 17 ulcerative colitis cases per 100000 person-years. The incidence of Crohn's disease in asthma and COPD patients was $27 \%$ and 55\% higher than in the general population of Québec; the incidence of ulcerative colitis was $30 \%$ higher among COPD patients compared to the general population.

Incidence of inflammatory bowel disease was significantly increased in asthma and COPD patients compared to the general population of Québec. Incidence rates were particularly high in patients with COPD. Awareness of an association between airway diseases and inflammatory bowel disease in older age groups may play an important role in earlier detection of bowel disease and in the therapeutic management of such patients.

@ERSpublications

Patients with airway diseases have a higher incidence of inflammatory bowel disease than the general population http://ow.ly/D6dXl

A press release for this article is available from www.erj.ersjournals.com/site/misc/presspack.xhtml

Received: April 282014 | Accepted after revision: Oct 072014 | First published online: Nov 182014

Support statement: Financial support for this study was provided through internal funding from McGill University Health Centre.

Conflict of interest: Disclosures can be found alongside the online version of this article at erj.ersjournals.com

Copyright OERS 2015 


\section{Introduction}

Inflammatory bowel disease (IBD) and airway diseases may be associated through common inflammatory pathways, genetic and environmental factors [1-4]. The intestinal and respiratory epithelia share the same embryological origin, have a similar anatomic structure and serve as organ barriers between the body and the environment. Immunological dysfunctions triggered by environmental factors are a common element in the pathogenesis of both IBD and airway diseases, such as asthma and chronic obstructive pulmonary disease (COPD). Furthermore, pulmonary involvement, in its multiple forms [5], is one of the extra-intestinal manifestations of IBD, while an increased intestinal permeability was described in asthmatic patients [6].

Epidemiological studies have shown that the prevalence of IBD was significantly increased among patients with airway diseases compared to prevalence in the general population $[2,7]$. Childhood asthma was also associated with increased risk of developing Crohn's disease [8]. However, the incidence of IBD among patients with airway diseases has never been assessed. Québec is the province with the second highest incidence of Crohn's disease in Canada [9, 10], with a prevalence of asthma and COPD above the Canadian average [11], and with the highest mortality rate due to COPD of all Canadian provinces [12]. We sought to assess whether the incidences of Crohn's disease and ulcerative colitis are increased in patients with airway diseases compared to the general population of Québec, Canada.

\section{Methods}

Data source

The administrative health databases of the Régie de l'assurance maladie du Québec (RAMQ) capture the physician visits, procedures, hospitalisations, patient demographics and pharmacy claims under the provincial public drug plan for all residents. The provincial pharmacy claims databases cover approximately $40 \%$ of Québec residents: all residents aged 65 years and older, those who receive social assistance, and those who do not have private drug insurance through their employer [13]. Data regarding type of drugs, quantity, date dispensed and duration, are missing or out of range in less than $0.4 \%$ of records [14]. Ethics approval for this study was granted by the research ethics committee of the Sir Mortimer B. Davis Jewish General Hospital, Montreal, QC, Canada.

\section{Study design and case identification}

In this population-based retrospective cohort study, the source population consisted of all subjects who, between January 1, 1990 and December 31, 2007, were dispensed at least one of several respiratory medications: any form of $\beta$-agonist, theophylline, ipratropium bromide, tiotropium, sodium cromoglycate, nedocromil, ketotifen, leukotriene antagonists (montelukast and zafirlukast) or inhaled corticosteroids (ICS). A base population of airway disease patients was identified from the source population as all subjects with three or more prescriptions for respiratory medication in any 1-year period and on at least two different occasions. Several studies addressing the risk of fractures, cataracts, glaucoma, tuberculosis and pneumonia among patients with airway disease have successfully employed the RAMQ pharmacy claims databases and the above mentioned criteria to identify patients with chronic airway disease [15-19].

From the base airway disease population we selected two mutually exclusive cohorts of asthma and COPD patients. The asthma cohort included all subjects from the base population aged 40 years or less at cohort entry (third prescription for respiratory medication). The COPD cohort included all subjects from the base population aged 41 years or older at cohort entry (third prescription for respiratory medication) who were never dispensed leukotriene antagonists (montelukast, zafirlukast), sodium cromoglycate, nedocromil or ketotifen and who never had a diagnostic code of asthma recorded in the database. The age of 40 years was used as cut-off point in other claims database studies related to COPD [20, 21].

Among patients with asthma and COPD we subsequently identified IBD cases using a case definition requiring at least four physician billings within a 2-year time period or one hospitalisation with a primary diagnosis of IBD (either Crohn's disease or ulcerative colitis). The IBD case definition was developed in Alberta, Canada, and was validated through chart review and linkage with endoscopy records [22]. The diagnosis codes used were 555.x and K50.xx for Crohn's disease and 556.x and K51.xx for ulcerative colitis, according to the ninth and 10th revisions of the International Classification of Diseases. The scoring system used to further classify IBD cases into Crohn's disease, ulcerative colitis or IBD not classified was described elsewhere [10].

\section{Crohn's disease and ulcerative colitis incidence}

Incident cases were defined as the individuals in the asthma and COPD cohort who met the case definition for Crohn's disease or ulcerative colitis during 2001-2006 and who had been free of IBD for at least 2 years prior to the beginning of the 2 -year case-defining period. The IBD-free period was not 
required for children 2 years of age or younger at diagnosis. We computed Crohn's disease and ulcerative colitis incidence in patients with asthma and COPD during 2001-2006 because information regarding incidence in the general population was also available from our previous work [10] for that period, and IBD cases were identified using the same methodology. Data regarding hospitalisations in 2007 were incomplete; therefore, incidence in 2007 was not assessed.

All cases were considered as diagnosed on the date they met the case definition, whether that occurred at the time of a hospitalisation with Crohn's disease or ulcerative colitis as the primary diagnosis or at the fourth physician billing with a Crohn's disease or ulcerative colitis diagnostic code.

\section{Statistical analysis}

The age- and sex-standardised incidence rates were calculated using the direct method and the 2001 Census population for the Québec province [23]. Data analysis was performed using the SAS/STAT software, version 9.3 of the SAS System for Windows, SAS Institute Inc., Cary, NC, USA. All graphical elements were produced using the $\mathrm{R}$ software, version 3.0.1, R Foundation for statistical computing, Vienna, Austria.

\section{Results}

From the base population, 136178 subjects were selected into the asthma cohort and 143904 into the COPD cohort. The characteristics of subjects with asthma and COPD who developed IBD during 20012006 are presented in table 1.

The mean \pm SD age at diagnosis in the asthma cohort was $26 \pm 11$ years for Crohn's disease and $31 \pm 10$ years for ulcerative colitis; in the COPD cohort the mean \pm SD age at diagnosis was $69 \pm 11$ years for Crohn's disease and $72 \pm 11$ years for ulcerative colitis. The mean \pm SD duration of follow-up before IBD diagnosis was $6.5 \pm 4$ years for the asthma cohort and 5.2 \pm 3.6 years for the COPD cohort.

\section{Crohn's disease and ulcerative colitis incidence}

The age-specific incidence rates of Crohn's disease and ulcerative colitis among patients with asthma and COPD are plotted in figure 1. In the asthma cohort the highest incidence for Crohn's disease, 34.3 cases per 100000 person-years, occurred in the 20-29-year-old age group; for ulcerative colitis, the highest incidence was 14.9 cases per 100000 person-years, in the 30-39-year-old group. In the COPD cohort the highest incidence of Crohn's disease, 35.5 cases per 100000 person-years, occurred in the 50-59-year-old age group; for ulcerative colitis, the highest incidence was 24.9 cases per 100000 person-years, in the 60-69-year-old group. The average Crohn's disease incidence rate for the 2001-2006 period was 23.07 cases

TABLE 1 Characteristics of subjects with asthma and chronic obstructive pulmonary disease (COPD) who developed inflammatory bowel disease (IBD) during 2001-2006

Asthma cohort

\section{IBD cases}

Age at IBD diagnosis

$0-9$ years

$10-19$ years

$20-29$ years

$30-39$ years

40-49 years

$50-59$ years

60-69 years

$70-79$ years

$80+$ years

Sex

Female

Male

Type of IBD

Crohn's disease

Ulcerative colitis

IBD unclassified ${ }^{\#}$
202

COPD cohort

$\begin{array}{cc}8(3.96) & \\ 50(24.75) & \\ 53(26.24) & \\ 55(27.23) & 7(4.96) \\ 35(17.33) & 19(13.48) \\ 1(0.5) & 33(23.4) \\ & 48(34.04) \\ & 34(24.11) \\ & 75(53.19) \\ 134(66.34) & 66(46.18) \\ 68(33.66) & \\ & 76(53.9) \\ 144(71.29) & 57(40.43) \\ 51(25.25) & 8(5.67) \\ 7(3.47) & \end{array}$

Data are presented as $\mathrm{n}$ or $\mathrm{n}(\%) .{ }^{\#}$ : fairly even mix of Crohn's disease and ulcerative colitis diagnostic codes. 

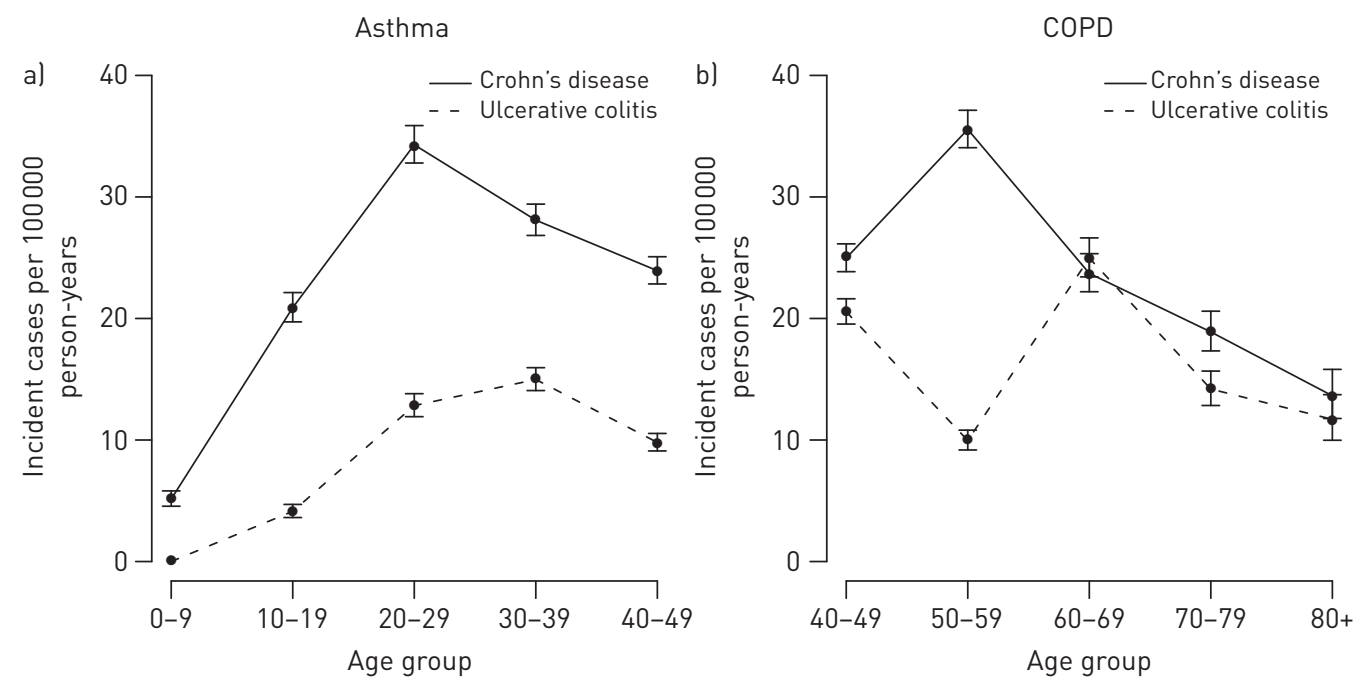

FIGURE 1 Age-specific incidence rate of inflammatory bowel disease in patients with a) asthma and b) chronic obstructive pulmonary disease (COPD), Québec, Canada, 2001-2006. For each value of age-specific incidence rate the vertical lines mark the limits of the upper and lower range of the $95 \%$ confidence interval.

per 100000 person-years in the asthma cohort and 26.17 cases per 100000 person-years in the COPD cohort. The average ulcerative colitis incidence was 8.8 cases per 100000 person-years in the asthma cohort and 17.04 cases per 100000 person-years in the COPD cohort, as shown in table 2.

The rate ratios showed that the incidence of Crohn's disease was higher than in the general population of Québec by $27 \%$ and $55 \%$ for asthma and COPD patients, respectively. The incidence of ulcerative colitis was $30 \%$ higher in COPD patients than in the general population. For Crohn's disease, the age-specific incidence in patients with asthma and COPD was significantly higher than in the general population for all age groups, with the exception of subjects aged 10-19 and over 70 years old. For ulcerative colitis we found a significantly higher incidence in asthma subjects aged 30-39 and COPD subjects aged 40-49 and 60-69 years compared to ulcerative colitis incidence for the same age groups in the general population.

The overall female/male ratio for Crohn's disease was 1.46 (95\% CI 1.39-1.53) in the asthma cohort and 2.15 (95\% CI 2.02-2.28) in the COPD cohort. For ulcerative colitis there was no significant difference

TABLE 2 Age-specific incidence of Crohn's disease and ulcerative colitis in patients with asthma and chronic obstructive pulmonary disease (COPD) and comparison with incidence rates in the Québec, Canada general population, 2001-2006

Age group/cohort at IBD diagnosis

Asthma
Overall
$0-9$ years
$10-19$ years
$20-29$ years
$30-39$ years
$40-49$ years
COPD

Overall

40-49 years

$50-59$ years

60-69 years

$70-79$ years

$80+$ years
Crohn's disease

Incidence rate

23.07
5.14
20.89
34.29
28.09
23.93

26.17
24.97
35.56
23.71
18.89
13.64

$1.27(1.22-1.31)$

$2.11(1.7-2.61)$

$1.05(0.97-1.15)$

$1.18(1.1-1.26)$

$1.42(1.32-1.53)$

$1.31(1.23-1.41)$

$1.55(1.49-1.62)$

$1.37(1.28-1.47)$

$2.18(2.02-2.36)$

$1.5(1.35-1.66)$

$1.1(0.97-1.24)$

$0.96(0.78-1.18)$
Ulcerative colitis

Incidence rate IRR $^{\#}$

\#: incidence rate ratio (with $95 \%$ confidence interval) between the incidence rate in the airway disease cohort and the incidence rate in the Québec population [10]. Statistically significant results in bold. 

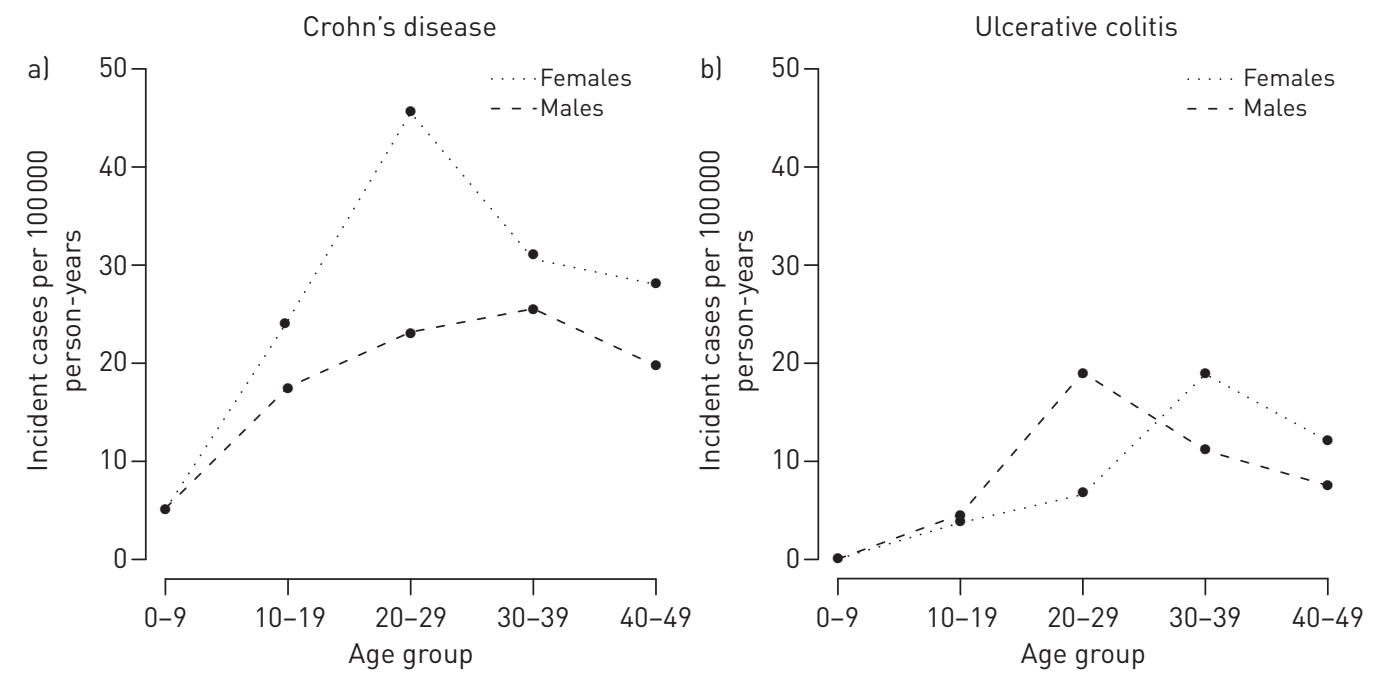

FIGURE 2 Age-specific inflammatory bowel disease incidence by sex in patients with asthma, Québec, Canada, 2001-2006.

between incidence rates in females and males in the asthma cohort but there were significantly more males in the COPD cohort, as figures 2 and 3 illustrate.

\section{Discussion}

In this administrative health database study we found that the incidences of both Crohn's disease and ulcerative colitis in patients with asthma and COPD were significantly higher compared to the general population of Québec during 2001-2006. Several European studies have reported similar findings in terms of IBD prevalence and the risk of developing $\operatorname{IBD}[2,7,8]$. Indeed, a retrospective analysis of outpatients with airway diseases in UK found up to a four-fold increase in IBD prevalence compared to the published local prevalence [7]. A study using the Swedish disease registries showed that the risk of developing IBD in patients with COPD was approximately double that in the controls without COPD, and that there was also a significant increase in the risk of IBD among first-degree relatives of COPD patients [2]. The underlying genetic, physiological and immune mechanisms that may explain the association between IBD and airway diseases were reviewed by KeELY et al. [24]. Regions of genetic linkage identified through genome-wide analyses associated with asthma, Crohn's disease and other autoimmune diseases were shown to overlap [25]. An impairment of intestinal permeability, which may play an important role in susceptibility to IBD [26], was described in subjects with both asthma [8] and COPD [27], further substantiating the association between these chronic inflammatory conditions.
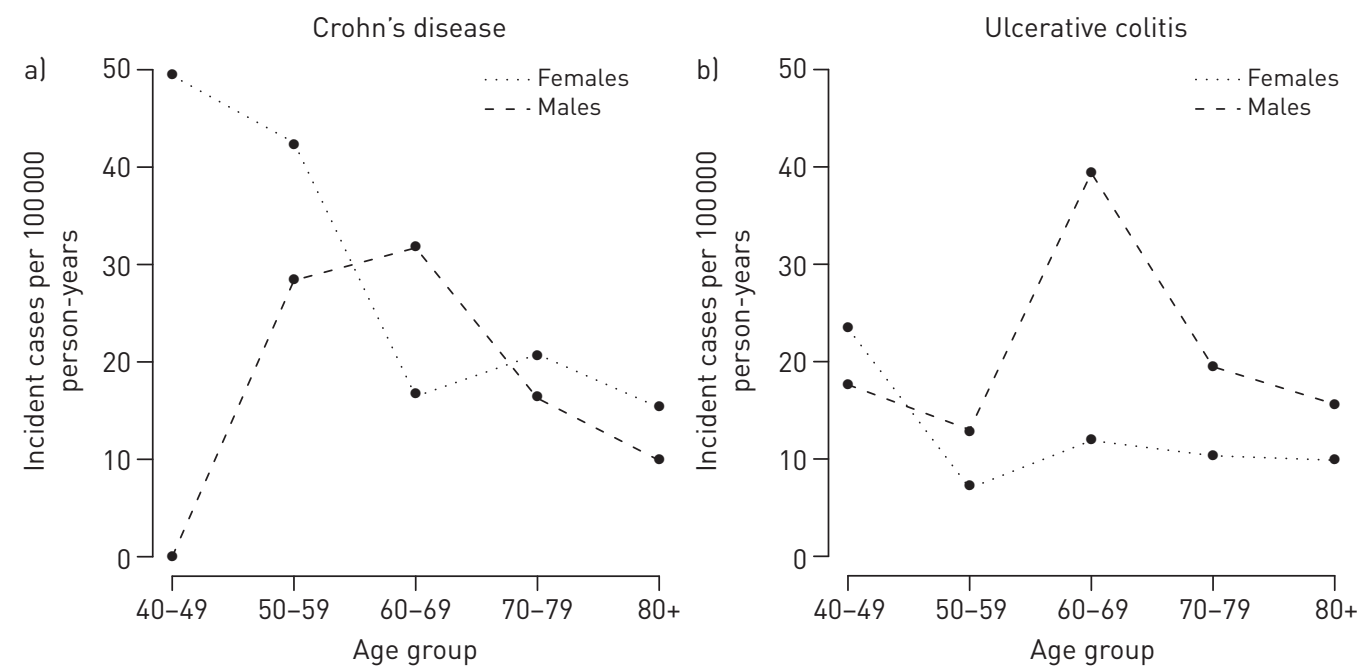

FIGURE 3 Age-specific inflammatory bowel disease incidence by sex in patients with chronic obstructive pulmonary disease, Québec, Canada, 2001-2006. 
In our study, the highest incidence rate ratio was observed in children up to 10 years old for the asthma cohort and in adults aged 50-59 years old for the COPD cohort, where the incidence of Crohn's disease was more than double that in the general population. The findings of VIRTA et al. [8] were also suggestive of an association between childhood asthma and Crohn's disease, while two other studies failed to find a significant association $[28,29]$.

Our results are suggestive of an association between COPD and incident IBD, as the incidence of both Crohn's disease and ulcerative colitis in elderly COPD patients was significantly higher than in the general population. The ulcerative colitis incidence reached 25 cases per 100000 person-years in the 60-69 years age group, $84 \%$ higher than in the general population.

We found that there was an overall predominance of female incident cases of Crohn's disease, and that the incidence of Crohn's disease in females for both asthma and COPD patients was significantly higher than in males for most age groups with the exception of children under the age of 10 and adults over 70 years old. We found no significant difference between the sexes in terms of incident ulcerative colitis cases in the asthma cohort. There was a significantly higher incidence of ulcerative colitis cases in males over the age of 50 years compared to females in the COPD cohort. Our findings are in agreement with the sex distribution of IBD in the general population $[9,10,30]$. The age-specific sex distribution in Crohn's disease incident cases and the predominance of female subjects (64\%) in the asthma cohort also reflect the higher number of female patients with asthma in Québec [11].

One of the limitations of our study is related to the use of prescription information to identify cases with airway disease, given that in Québec about $40 \%$ of the population, including all residents over the age of 65 years, is covered by the public drug plan. This may have an impact on the generalisability of our findings regarding the association between asthma and incident Crohn's disease in children and young adults. Further studies are needed to confirm such an association in other provinces or jurisdictions where prescription information is available for all age groups. However, given that all prescriptions for residents aged 65 years and older are part of the public drug plan, the use of prescription information will have little impact on the generalisability of our results regarding the association between COPD and incident IBD in seniors.

Another factor that may limit the generalisability of the findings is the genetic mix of the Québec population resulting from immigration. A study addressing the epidemiology of Crohn's disease in Québec has shown that a proportion of immigrants above $10 \%$ in health-related geographical divisions was associated with a significantly lower incidence of Crohn's disease; in contrast, a proportion of Jewish descendants above $20 \%$ was associated with a significantly increased incidence of Crohn's disease [9]. Further studies in other geographical locations may be needed to confirm such findings.

Another limitation stems from the lack of information regarding smoking, a common limitation in studies based on administrative health data. Tobacco smoking is a known risk factor for both COPD and Crohn's disease, and a protective factor for ulcerative colitis [31], and it may, in part, explain the association between these diseases. However, Еквом et al. [2] found that the risk of Crohn's disease in COPD patients was higher than the risk attributable to smoking alone, and that the risk of ulcerative colitis was also increased. If the association between COPD and IBD would be due entirely to the confounding effect of smoking, then the number of ulcerative colitis cases in patients with COPD should be small. Our results show a significantly higher incidence of ulcerative colitis among patients with COPD aged 60 years and older. Therefore, we believe it is unlikely that smoking alone could have accounted for the increased number of incident IBD cases.

To date, this is the first population-based study to investigate the association between airway diseases and incidence of IBD. The identification of airway disease patients through prescriptions for specific respiratory medication was shown to be valid and was used in many administrative health database studies $[15,16,18,19]$. The incidence of IBD in asthma and COPD patients was assessed using the same methodological approach as in our previous work addressing IBD incidence in the general population of Québec, therefore insuring direct comparability of results by age, sex and time period [10].

In conclusion, the incidences of both Crohn's disease and ulcerative colitis were significantly increased in asthma and COPD patients during 2001-2006 compared to the general population of Québec. Women with asthma were more likely to develop Crohn's disease than men, and men with COPD were more likely to develop ulcerative colitis than women. Confirmation of such results in future studies may have implications in earlier detection of IBD and in the therapeutic management of patients. Medical professionals involved in the care of airway disease patients who develop digestive symptoms need to be aware of the possible occurrence of new cases of IBD, even in older age groups and regardless of smoking status. 


\section{References}

1 Bernstein CN, Wajda A, Blanchard JF. The clustering of other chronic inflammatory diseases in inflammatory bowel disease: a population-based study. Gastroenterology 2005; 129: 827-836.

2 Ekbom A, Brandt L, Granath F, et al. Increased risk of both ulcerative colitis and Crohn's disease in a population suffering from COPD. Lung 2008; 186: 167-172.

3 Hemminki K, Li X, Sundquist J, et al. Subsequent autoimmune or related disease in asthma patients: clustering of diseases or medical care?. Ann Epidemiol 2010; 20: 217-222.

4 Weng X, Liu L, Barcellos LF, et al. Clustering of inflammatory bowel disease with immune mediated diseases among members of a northern California-managed care organization. Am J Gastroenterol 2007; 102: 1429-1435.

5 Tzanakis NE, Tsiligianni IG, Siafakas NM. Pulmonary involvement and allergic disorders in inflammatory bowel disease. World J Gastroenterol 2010; 16: 299-305.

6 Benard A, Desreumeaux P, Huglo D, et al. Increased intestinal permeability in bronchial asthma. J Allergy Clin Immunol 1996; 97: 1173-1178.

7 Raj AA, Birring SS, Green R, et al. Prevalence of inflammatory bowel disease in patients with airways disease. Respir Med 2008; 102: 780-785.

8 Virta LJ, Ashorn M, Kolho KL, et al. Cow's milk allergy, asthma, and pediatric IBD. J Pediatr Gastroenterol Nutr 2013; 56: 649-651.

9 Lowe AM, Roy PO, Bigras-Poulin M, et al. Epidemiology of Crohn's disease in Quebec, Canada. Inflamm Bowel Dis 2009; 15: 429-435.

10 Bitton A, Vutcovici M, Patenaude V, et al. Epidemiology of inflammatory bowel disease in Quebec: recent trends. Inflamm Bowel Dis 2014; 20: 1770-1776.

11 Statistics Canada. Canadian Community Health Survey - Health Indicator Profile, Annual Estimates, by Age Group and Sex, Canada, Provinces, Territories, Health Regions (2012 boundaries) and Peer Groups (Table 105-0502), 2012. www23.statcan.gc.ca/imdb/p3Variable.pl?Function=getStatDEDataList\&DEItem_Id=62231\&DECItem_Id= 379\&Repclass $=591 \&$ SurvId $=3226 \&$ SurvVer $=2 \& I n s t a I d=15282 \& I n s t a V e r=10 \& \mathrm{t}=\mathrm{t} \& \mathrm{db}=\mathrm{imdb}$ Date last updated: December 2, 2013.

12 Canadian Lung Association. Chronic Obstructive Pulmonary Disease (COPD). A National Report Card. Ottawa, Canadian Lung Association, 2005.

13 Pomey MP, Forest PG, Palley HA, et al. Public/private partnerships for prescription drug coverage: policy formulation and outcomes in Quebec's universal drug insurance program, with comparisons to the Medicare prescription drug program in the United States. The Milbank Quarterly 2007; 85: 469-498.

14 Tamblyn R, Lavoie G, Petrella L, et al. The use of prescription claims databases in pharmacoepidemiological research: the accuracy and comprehensiveness of the prescription claims database in Québec. J Clin Epidemiol 1995; 48: 999-1009.

15 Brassard P, Suissa S, Kezouh A, et al. Inhaled corticosteroids and risk of tuberculosis in patients with respiratory diseases. Am J Respir Crit Care Med 2011; 183: 675-678.

16 Ernst P, Baltzan M, Deschênes J, et al. Low-dose inhaled and nasal corticosteroid use and the risk of cataracts. Eur Respir J 2006; 27: 1168-1174.

17 Ernst P, Gonzalez AV, Brassard P, et al. Inhaled corticosteroid use in chronic obstructive pulmonary disease and the risk of hospitalization for pneumonia. Am J Respir Crit Care Med 2007; 176: 162-166.

18 Gonzalez AV, Li G, Suissa S, et al. Risk of glaucoma in elderly patients treated with inhaled corticosteroids for chronic airflow obstruction. Pulm Pharmacol Ther 2010; 23: 65-70.

19 Suissa S, Baltzan M, Kremer R, et al. Inhaled and nasal corticosteroid use and the risk of fracture. Am J Respir Crit Care Med 2004; 169: 83-88.

20 Hasegawa K, Tsugawa Y, Tsai CL, et al. Frequent utilization of the emergency department for acute exacerbation of chronic obstructive pulmonary disease. Respir Res 2014; 15: 40.

21 Marton JP, Boulanger L, Friedman M, et al. Assessing the costs of chronic obstructive pulmonary disease: the state medicaid perspective. Respir Med 2006; 100: 996-1005.

22 Rezaie A, Quan H, Fedorak RN, et al. Development and validation of an administrative case definition for inflammatory bowel diseases. Can J Gastroenterol 2012; 26: 711-717.

23 Statistics Canada. Age and Sex for Population, for Canada, Provinces, Territories and Federal Electoral Districts. www12.statcan.gc.ca/english/census01/products/standard/themes/Rp-eng.cfm?TABID=1\&LANG=E\&A=R\&APATH= 3\&DETAIL $=1 \& \mathrm{DIM}=0 \& \mathrm{FL}=\mathrm{A} \& \mathrm{FREE}=0 \& \mathrm{GC}=24 \& \mathrm{GID}=361266 \& \mathrm{GK}=1 \& \mathrm{GRP}=1 \& \mathrm{O}=\mathrm{D} \& \mathrm{PID}=55436 \& \mathrm{PRID}=0$ \& PTYPE $=55430,53293,55440,55496,71090 \& S=0 \& S H O W A L L=0 \& S U B=0 \&$ Temporal $=2001 \&$ THEME $=37 \&$ VID $=0 \&$ VNAMEE $=\& \mathrm{VNAMEF}=\& \mathrm{D} 1=0 \& \mathrm{D} 2=0 \& \mathrm{D} 3=0 \& \mathrm{D} 4=0 \& \mathrm{D} 5=0 \& \mathrm{D} 6=0$ Date last updated: August 15, 2012.

24 Keely S, Talley NJ, Hansbro PM. Pulmonary-intestinal cross-talk in mucosal inflammatory disease. Mucosal Immunol 2012; 5: 7-18.

25 Becker KG, Simon RM, Bailey-Wilson JE, et al. Clustering of non-major histocompatibility complex susceptibility candidate loci in human autoimmune diseases. Proc Nat Acad Sci USA 1998; 95: 9979-9984.

26 Schreiber S, Rosenstiel P, Albrecht M, et al. Genetics of Crohn disease, an archetypal inflammatory barrier disease. Nat Rev Genet 2005; 6: 376-388.

27 Rutten EP, Lenaerts K, Buurman WA, et al. Disturbed intestinal integrity in patients with COPD: effects of activities of daily living. Chest 2014; 145: 245-252.

28 Yun HD, Knoebel E, Fenta Y, et al. Asthma and proinflammatory conditions: a population-based retrospective matched cohort study. Mayo Clin Proc 2012; 87: 953-960.

29 Juhn YJ. Influence of asthma epidemiology on the risk for other diseases. Allergy Asthma Immunol Res 2012; 4: 122-131.

30 Bernstein CN, Wajda A, Svenson LW, et al. The epidemiology of inflammatory bowel disease in Canada: a population-based study. Am J Gastroenterol 2006; 101: 1559-1568.

31 Birrenbach T, Böcker U. Inflammatory bowel disease and smoking. A review of epidemiology, pathophysiology, and therapeutic implications. Inflamm Bowel Dis 2004; 10: 848-859. 